\author{
Proceedings of the $10^{\text {th }}$ International Conference on Applied Informatics \\ Eger, Hungary, January 30-February 1, 2017. pp. 171-178 \\ doi: 10.14794/ICAI.10.2017.171
}

\title{
Energy consumption of Geographic Routing Mechanisms in Wireless Sensor Networks - a Survey
}

\author{
Mohamed Amine Korteby, Zoltán Gál \\ University of Debrecen \\ m.a.korteby@gmail.com \\ Gal.Zoltan@inf.unideb.hu
}

\begin{abstract}
Geographic routing algorithms have received an increasing interest as a routing solution for WSN over the past years. These algorithms refer to nodes by their location, rather than their address and use this coordinates to route greedily when possible toward the destination. These tasks are executed for a better scalability, successful data transmission and lower energy consumption. Most geographic routing mechanisms tend to have a limitation regarding routing toward dead ends and obstacles. Some algorithms were developed for packet radio and wired networks as a greedy strategy, based on the relative direction of the source and destination. Instead of selection the next hop by the minimum criteria, two hops information and heuristic model are used to avoid congested areas and void regions. To increase the delivery rate, multipath strategy algorithms were introduced to broadcast a message through different nodes that are within an incomplete pyramid determined by the source and destination nodes. On the other hand, other techniques tend to not rely on location information but define a scalable coordinate based routing algorithm that involve assigning virtual coordination to each node. These techniques are powerful and reliable but indeed they fail to have good packet delivery, guaranty transmission to all target nodes and working on large void areas. These mechanisms do not focus on energy consumption and network life time. Hence the need of a new approach that concerns energy efficiency, greatly shorten the average data transmission and prolong the network lifespan. In our paper, a survey of existing geographic routing mechanisms and a new routing solution in wireless sensor network environment, named ALBEMS will be presented.
\end{abstract}

Keywords: geographic routing, virtual coordinates, wireless sensor network, energy efficiency.

MSC: 90B18, 68M10, 68M12 


\section{Introduction}

Wireless sensor networks have change our way to collect scientific data and acknowledging our environment. Recent advances in wireless and mobile electronic communication technology has led to increased attention to wireless ad-hoc and sensor networks. Numerous position-based routing algorithms have been proposed to satisfy the demands of particular networks and real applications. The paper has following structure. In chapter 2 eight location-based routing protocols for WSN are presented. Our routing proposal is described in the chapter 3 including the architecture and some aspects of the signaling and data communication mechanism. In chapter 4 we conclude and we give the necessary continuation of the proposed new mechanism.

\section{Location-Based Protocols for WSNs}

\subsection{Trajectory-based forwarding (TBF)}

Trajectory-based forwarding (TBF) requires a dense network and a coordinate system (GPS), for node positioning and neighbors distance estimation. To send data, the source encodes the path and embeds it into each packet. Intermediate nodes decode the path and perform a greedy forwarding method for the packet to follow the same encoded route. TBF implement a multipath routing strategy to increase the reliability and network capacity. This mechanism is not affected by sensor mobility as the source path does not include the id of the next hope node $[1]$.

\subsection{Bounded Voronoi greedy forwarding (BVGF)}

BVGF is a localized routing protocol that makes greedy routing decisions based on one-hop neighbor locations. When node i, Vor(i) needs to forward a packet, a neighbor node $\mathrm{j}$, Vor $(\mathrm{j})$ is eligible as the next hop only if the line segment joining the source and the destination intersects $\operatorname{Vor}(\mathrm{j})$ or coincides with one of the boundaries of $\operatorname{Vor}(\mathrm{j})$. BVGF chooses as the next hop the neighbor that has the shortest Euclidean distance to the destination among all eligible neighbors. When there are multiple eligible neighbors that are close to the destination, the forwarding node randomly chooses one among them as the next hop. It does not help the sensors deplete their battery power uniformly. Each sensor actually has only one next hop to forward its data to the destination. Therefore, any data dissemination path between a source and destination will always have the same chain of the next hops, which will severely suffer from battery power consumption. BVGF does not consider energy as a metric [2]. 


\subsection{Geographic random forwarding (GeRaF)}

Geographic random forwarding $(\mathrm{GeRaF})$ is a location-based routing protocol with an awake/sleep scheduling scheme where the nodes periodically switch from an active and asleep state using a given duty cycle [3]. If a given node wants to send data, it becomes active and broadcast a packet. This broadcast packet contains the source and destination location address. Thus, the nodes in the network do not store any neighbors' information and duty cycle awake/sleep scheduling. The zone facing the destination is called the forwarding area and is divided into priority area, the intermediate node is chosen from the highest priority area. A situation can happen when all the nodes inside the highest priority region are in sleep mode, in order the solve the problem, second highest priority intermediate node is chosen in the next transmission attempt. This attempt will be repeated until a maximum number of retries is reached.

\subsection{An Energy- Efficient Coordination Algorithm for Topol- ogy Maintenance (SPAN)}

SPAN is a proactive power saving geographic routing protocol for multi-hop ad ho wireless networks, where each node enters all the information it receives in broadcast updates into a neighbor table. Span main idea is to elect a coordinator from all nodes in the network adaptively to provide an equal global connectivity, that coordinator election reduces energy consumption without significantly diminishing the capacity or connectivity of the network. The node elected as coordinator stay awake continuously and perform multi-hop packet routing within the ad hoc network, while other nodes remain in power-saving mode and periodically check if they should wake up and become a coordinator [4]. The results show that Span does not significantly degrade network capacity and can forward more packets under high load. Also as density increases, the energy saving is predicted to be high, however in practice, the energy saving was not found to be significant.

\subsection{Energy Conditioned Mean Square Error Algorithm}

The authors in [5] propose an energy conditioned mean square error algorithm (ECMSE) for WSN in need of efficient, location error-coping geographic routing. The retransmissions number are limited and the sensor nodes use low power transmission. The algorithm is designed for quality of service and high delivery rate without compromising the energy efficiency. ECMSE is the extension of the work in [6] which is a similar energy-optimal forwarding for error-robust mechanism (CMSER) and took the energy model of the work in [7]. ECMSE uses the statistical assumption of Gaussianly distributed location error and the energy coast of the forwarding decision. This mechanism is suited for large scale network where it gives better results in terms of overall energy consumption. 


\subsection{Modified Geographical Energy-Aware Routing Protocol in Wireless Sensor Networks (MGEAR)}

MGEAR is an energy efficient geographical routing protocol that uses gateway nodes and cluster heads to minimize the energy consumption of the sensor nodes. The gateway nodes collect the data form nodes and form the cluster heads, then the data is aggregated and transmitted to the base station. To do so, the network is divided into four distinguished logical regions, two of them use direct communication while the two-other part use clustering hierarchy, depending on probability and the residual energy of nodes [8]. The authors claim that MGEAR gives the largest network lifetime because of network division into logical part, while LEACH (Low-energy Adaptive Clustering Hierarchy) nodes die quickly.

\subsection{Anonymous Location-based Efficient Routing Protocol (ALERT)}

ALERT works on mobile scenario (moving sources and/or destination), it is also distinguished by its low cost and anonymity protection for resources, destination, and routes. Sensor nodes are randomly deployed in a rectangle area, at each step sources hierarchically partition the network into difference zones alternatively into horizontal and vertical direction until they are in the same zone as the destination. Relay nodes are chosen randomly in each step, which will generate an unpredictable routing path for each message. In addition, ALERT has a strategy to hide the data initiator among several initiators to strengthen the anonymity protection of the source [9].

\subsection{Location-Based Routing Protocol (LBRP)}

The LBRP works on mobile scenario, it uses an adaptive transmission power algorithm and greedy forwarding when routing the packets to save energy and enhancing the network lifetime. The algorithm uses a Location Service Module (LSM), this module needs to be triggered whenever a node has a packet to send [10]. The main purpose of using LMS is to provide the location of the next hop, the destination location and keeping track of the network nodes' position. A source node forwarding a packet adds the destination location in the packet for the intermediate node to forward the packet correctly. The intermediate nodes will extract the destination location from the packet and updates it if a new destination position is known, otherwise, the packet is sent without any changes. To determine the last position of the destination LSM periodically broadcast a beacon message that contains a sequence number. High sequence number means that the destination location is recent [10]. The authors claim that LBRP consumes less energy during data transmission, extend battery life and increases the sensor network availability. LBRP was never tested in real life scenario.

In Table 1 we compared Location-based protocols from the mobility, energy 
efficiency, aggregation level, QoS, scalability and multipath capability aspects.

\begin{tabular}{|c|c|c|c|c|c|c|}
\hline Protocoles & Mobility & Energy & Aggregation & QoS & Scalability & Multipath \\
\hline TBF & No & No & No & No & Limited & No \\
BVGF & No & Yes & No & No & Good & No \\
GERAF & No & Yes & No & No & Good & No \\
SPAN & No & Yes & No & No & Good & Yes \\
MGEAR & No & Yes & No & No & Good & No \\
ECMSE & No & Yes & Yes & Yes & Excellent & No \\
ALERT & Yes & Yes & No & No & Limited & Yes \\
LBRP & Yes & Yes & No & No & Limited & No \\
\hline
\end{tabular}

Table 1: Comparision of Location-based Routing Protocoles

\section{Architecture and running of the ALBEMS system}

The ALBEMS (Adaptive Location Based Emergency Message Service) system architecture is presented in Figure 1. The data is hierarchically forwarded through different levels of the system, which is composed of ALBEMS Nodes (AN). An AN node has one or several sensors which acquire information about a given phenomenon and send the data to the ALBEMS Sink Node (ASN). The ASN in this network architecture is in charge for forwarding the information to ALBEMS Operator $(\mathrm{AO})$. The $\mathrm{AO}$ center is responsible for processing the information captured by the AN nodes. The collected sensor data is passed from the AN to the ASN using a new proposed solution named ALBEMS Routing System (ARS), described in more details in section 3.

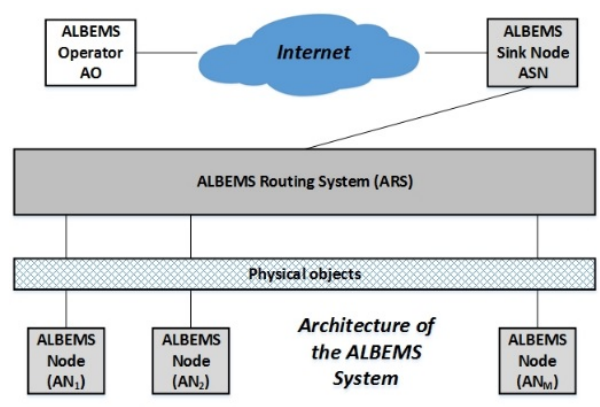

Figure 1: Architecture of the ALBEMS system

The AN nodes are placed in virtual coordinates known as Grid Points (GP). Filled Grid Points (FGP) have sensor nodes in their vicinity, defined by a sphere $\mathrm{G}(\mathrm{r}, \mathrm{V})$, where $\mathrm{r}$ is the radius and $\mathrm{V}$ is the Descartes coordinates of the sphere's 
center. The optimum value of $\mathrm{r}$ is a subject of new research task, not included in this paper (see Figure 2).

The initial value of $\mathrm{r}$ was considered $5 \%$ of the coverage radius of the radio interface. $\mathrm{Vx}, \mathrm{Vy}, \mathrm{Vz}$ are the virtual coordinates of GP. Because of the relatively small value of the $r$, vicinity radius, at most only one node can be in any $\mathrm{G}(\mathrm{r}, \mathrm{V})$. Non-Filled Grid Points (nFGP) have no sensor nodes in their vicinity.

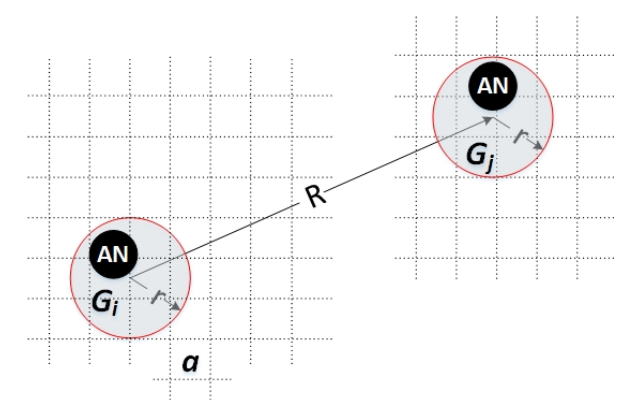

Figure 2: Relation between Grid points and AN nodes

The radio interface is able to cover distance at least $\mathrm{R}$. Four running modes have been defined for the sensor nodes to improve the energy efficiency of the network. The activities in these running modes are presented in Table 2. The GP can be classified as follow: AF, PF, AS for FGP; PS and obstacles are nFGP.

\begin{tabular}{|c|c|c|c|}
\hline Running mode & Data forwarding & Setup initiation & Signal forwarding \\
\hline Active Forwarder (AF) & + & + & + \\
Passive Forwarder (PF) & - & + & + \\
Active Sleeping (AS) & - & - & + \\
Passive Sleeping (PS) & - & - & - \\
\hline
\end{tabular}

Table 2: Running modes of the ALBEMS node

The First-Class Neighbors (FCN) are the nodes inside the intersection of the spherical sensing range $[R, R+d R]$ and the incomplete pyramid given in Figure 3. Because of the energy constraints we want our network to have the minimum number of hops as possible. Not to forget that having a large radius consume energy and having a small one leads to data latency. We defined an incomplete $3 \mathrm{D}$ shape pyramid having hexagon bases. This $3 \mathrm{D}$ shape is perfect over square or triangular shapes in our architecture because we want to cover the entire area without overlapping (i.e. we can cover the entire geographical region without any gaps), as we want to connect every existing sensor in the 3D space to reach our objective.

The proposed routing protocol is divided into three specific phases: i) Path Setup phase (PS_Phase); ii) Data Transmission phase (DT_Phase); iii) Path Release phase ( $\mathrm{PR} \_$Phase). The $\mathrm{PS} \_$Phase aims to discover multiple routes where 


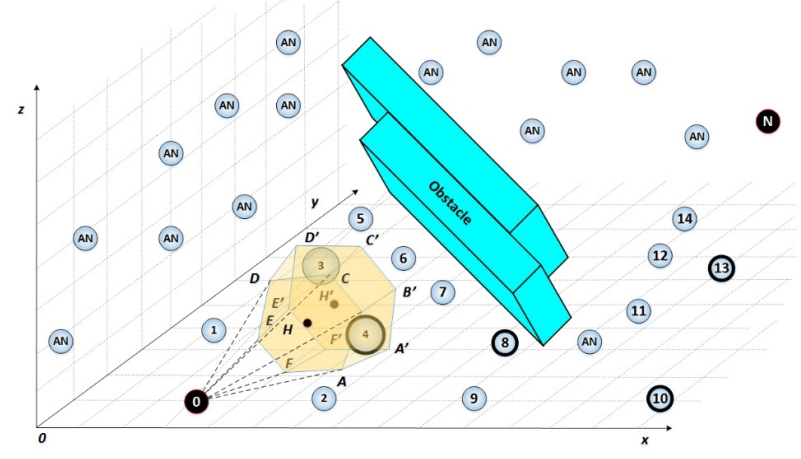

Figure 3: Routing mechanism of the ALBEMS system

each node performing a handshake with it FCN. The optimum path, in number of hops and remaining energy, is selected for the DT_Phase. In PR_Phase the network resources are freed for later connections.

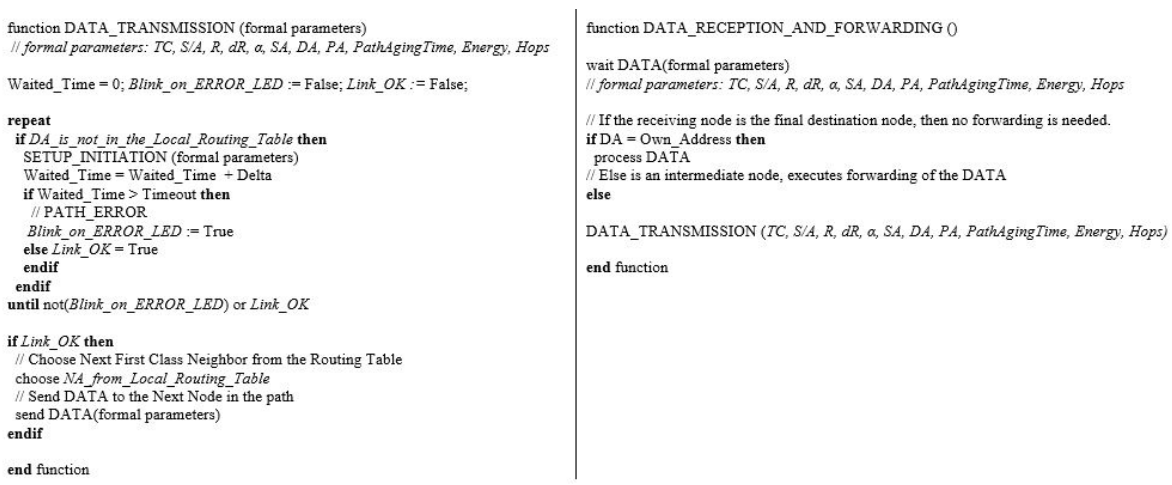

Figure 4: Data transmission, reception and forwarding mechanisms of the ALBEMS system

Any ALBEMS node which has message to send and has no matching routing record for the destination address, executes setup function to the destination. The PS_Phase is limited in time. Path error is when no acknowledgement is received from any node in the forwarding zone. After the successfully executed PS_Phase each node on the path receiving a message checks the local routing table for destination address and forwards the message (see Figure 4). 


\section{Conclusion}

In our paper we compared the most popular location based routing mechanisms used for wireless sensor networks. Only a small part of the existing such protocols have mobility, aggregation and QoS features integrated. The scalability and the mobility are inverse correlated characteristics for these mechanisms. Because of the connection oriented feature, the new geographic routing mechanism named ALBEMS, proposed by us in this paper, has more favorable features, like energy usage and QoS than the existing other WSN protocols. More analysis is required to highlight the specific features of this new geographic routing mechanism.

\section{References}

[1] Yuksel, M., Pradhan, R., Kalyanaraman, S., TBF: Trajectory-Based Forwarding Mechanisms for Ad-HOC Networks, Wireless Networks. Vol. 8 (2002), 481-494.

[2] Xing, G., Lu, C., Pless, R., Hunang, Q., On Greedy Geographic Routing Algorithms in Sensing-Covered Networks, Proceedings of the 5th ACM International Symposium on Mobile Ad Hoc Networking and Computing, Tokyo., (2004), 24-26.

[3] Zorzi, M. And Rao, R. , Geographic Random Forwarding (GeRaF) for Ad Hoc and Sensor Networks: Multihop Performance, IEEE Transaction on Mobile Computing. Vol. 2 (2003), 337-348.

[4] Chen, B., Jamieson, K., Balakrishnan, H. and Morris, R. , Span: An EnergyEfficient Coordination Algorithm for Topology Maintenance in Ad-Hoc Wireless Networks, Wireless Networks Vol. 8 (2002), 481-494.

[5] Popescu, A.M., Salman, N. And Kemp, A.H. , Energy Efficient Geographic Routing Robust Against Location Errors, IEEE Sensors Journal Vol. 14 (2014), 1944-1951.

[6] Popescu, A.M., Salman, N. And Kemp, A.H. , Geographic Routing Resilient to Location Errors, IEEE Wireless Communications Letters Vol. 2 (2013), 203-206.

[7] Peng, B. And Kemp, A.H. , Energy-Efficient Geographic Routing in the Presence of Localization Errors, Computer Networks Vol. 55 (2011), 856-872.

[8] Singh, P.K., Prajapati, A.K., Singh, A. And Singh, R.K. , Modified Geographical Energy-Aware Routing Protocol in Wireless Sensor Networks, International Conference on Emerging Trends in Electrical Electronics 85 Sustainable Energy Systems (ICETEESES) (2016), 208-212.

[9] Shen, H. and Zhao, L. , ALERT: An Anonymous Location-Based Efficient Routing Protocol in MANET's, IEEE Transactions on Mobile Computing Vol. 12 (2013), 1079-1093.

[10] Sammut, E. And Debono, C.J. , A Location-Based Routing Algorithm for Wireless Sensor Networks, Proceedings of IEEE International Conference on Computer as a Tool (EUROCON'15) (2015), 1-5. 